\title{
EVALUACIÓN PRELIMINAR DE DIFERENTES GERMOPLASMAS DE ARROZ (Oryza sativa) PARA DETER- MINAR LA PREFERENCIA DEL ÁCARO Steneotarsonemus spinki (tarsonemidae) EN COSTA RICA.
}

\author{
Ruth León González'
}

\begin{abstract}
RESUMEN
El estudio se realizó en la Región Chorotega y en la Región Pacífico Central. Las características de la Región Chorotega la ubican a una altura de $20 \mathrm{msnm}$, con una temperatura promedio de $27,85^{\circ} \mathrm{C}$, precipitación de $152,86 \mathrm{~mm}$, humedad relativa de $77,67 \%$., Se clasifica como zona de vida tipo Bosque Tropical Seco y la Región Pacífico Central, se encuentra a una altitud de $10 \mathrm{msnm}$, un promedio de $328 \mathrm{~mm}$ de precipitación, $27^{\circ} \mathrm{C}$ de temperatura y $77 \%$ de humedad relativa, se encuentra en la zona de vida, Bosque Tropical Húmedo. Se evaluaron variedades criollas (multiplicación y preservación de semillas) y materiales de rendimiento (líneas que se convertirán en variedades). Los datos de las líneas se promediaron y agruparon, basados en la preferencia o no del ácaro. Se formaron cuatro grupos con su respectiva escala y descripción. El comportamiento de $S$. spinki en las variedades criollas indicó que la variedad con más huevos fue Rex Oro (309) seguida de Chin Chin (275) y las que no presentaron huevos fueron las variedades Oryzica turipana, Miravalles, Blue Bonnet, CR-1821 y CR-4477. En cuanto a inmaduros la variedad más susceptible fue Chin Chin y las que no tuvieron fueron Oryzica turipana, Miravalles, Blue Bonnet, CR-1821, CR-4477, Tenorio y Gallote. En cuanto a la presencia de adultos la más susceptible fue Chin Chin y las que no se infestaron fueron Oryzica turipana, Miravalles, Blue Bonnet, CR-1821, CR-4477 y la Orosí. Las líneas de mayor rendimiento en la Región Chorotega fueron la CT15671-15-4-5-1-1-M con 5,8 t/ ha y obtuvo un promedio de 278 ácaros/ planta (a/ p) , CT15672-12-1-5-2-4-M con $5.6 \mathrm{t} /$ ha y con $96 \mathrm{a} / \mathrm{p}$, CT15765-13-7-4-2-1-M con 4,7 t/ ha y 0 ácaros, CT15675-7-1-7-3-2-M con 2 ácaros/ planta y CT15672-12-1-1-2-3-M con 4,0 t/ ha con 0 a/ p. En la Región Pacífico Central, los materiales de mayores rendimientos de campo fueron CT15675-7-1-4-2-1-M con 7,7 t/ ha, además no presentó durante las evaluaciones la presencia del ácaro, seguido de CT15765-18-1-5-3-2-M con 6,9 t/ ha, con cero ácaros, CT15672-12-1-2-1-1-M, con un promedio de 10,5 a/ p. No se encontró relación entre la población y el rendimiento ya que las líneas y variedades más infestadas fueron las más productivas. Por lo resultados preliminares de esta investigación es posible que la etapa fenológica, el manejo del cultivo y el clima influyeron en la población y afectaron el rendimiento.
\end{abstract}

Palabras claves: Germoplasma, arroz, variedades, líneas Oryza sativa, Steneotarsonemus spinki, Tarsonemidae.

\footnotetext{
${ }^{1}$ Instituto de Innovación y Transferencia de Tecnología Agropecuaria. E-mail: rleon@ inta.go.cr. Teléfono: (506)2231-5055;
} Telefax: (506)2231-5004. 


\section{INTRODUCCIÓN}

El cultivo del arroz (Oryza sativa L.), es la base fundamental de la dieta de los costarricenses. Se cultiva en las zonas bajas, desde el nivel del mar hasta $850 \mathrm{msnm}$, siendo las principales zonas productoras de arroz la región Brunca, Chorotega, Huetar Norte y Pacífico Central (MAG 1983). El arroz contiene naturalmente apreciables cantidades de tiamina, riboflavina y niacina, así como fósforo, hierro y potasio, lo que lo hace ser, el sustento principal de la población mundial desde hace miles de años; es uno de los alimentos más versátiles que existen (FAO 2004).

Cada variedad posee sus características fenotípicas y genotípicas que las hacen preferidas por los agricultores, así como por la producción y resistencia a plagas y enfermedades. Por ejemplo, CR-1113 se caracteriza por ser una variedad de paja corta, de hojas erectas, tallos gruesos, de buen macollamiento, resistente al acame, que florece entre 95 y 100 días después de la siembra y dura a la cosecha entre 127 135 días, es resistente a la mayoría de las enfermedades que afectan el cultivo, pero susceptible a piricularia (MAG 1991) y al ácaro del vaneo (León 2004), mientras que CR-5272 es una variedad de porte bajo, hojas erectas, macollamiento moderado, resistente al acame, con floración entre los 80 y 85 días después de la siembra y una duración a la cosecha entre 110 y 115 días (MAG 1991).

Las parcelas de observación tienen como objetivo, evaluar a nivel nacional material genético altamente promisorio, que será utilizado posteriormente como materiales de rendimiento en experimentos regionales, como un paso previo a la liberación de nuevas variedades, de ahí que cada parcela corresponde a una variedad, se evaluaron las variables agronómicas: patológicas, rendimiento, calidad molinera y culinaria.

El cultivo se relaciona con diversos ácaros. Estos afectan al cultivo por el daño al follaje y la pérdida económica, ya que además, reducen la cantidad de granos, y por lo tanto se recurre a la aplicación de insecticidas y acaricidas de origen químico lo que aumenta los costos de producción y contaminación al ambiente. Algunos estudiosos del cultivo, consideran que el ácaro del vaneo se encuentra en Costa Rica desde hace siete años, pero no es sino hasta el año 2004 que se reporta en las plantaciones de arroz.

Los ácaros asociados al cultivo de arroz en Costa Rica son: Oligonychus pratensis (Banks)* $^{*}$ Schizotetramychus freitezi Ochoa \& Von Lindeman*, Schizotetramychus pseudolycurus Ochoa \& Von Lindeman* (Acari: Tetranychidae) ( ${ }^{\star}$ Ochoa y Aguilar, 1991); cabe destacar que éstos ácaros, normalmente se presentan en forma esporádica y en bajas poblaciones, por lo que se hace necesario el muestreo para determinar las densidades que ameritan una decisión de combate. Sin embargo, el ácaro Steneotarsonemus spinki Smiley (Acari: Tarsonemidae), ha tomado características de plaga ya que además de las altas poblaciones, beneficia el desarrollo de un complejo de enfermedades fungobacteriales por lo que actualmente se le considera la plaga de mayor importancia económica en el cultivo del arroz en el país.

ElÁcarodelVaneodelArroz(Steneotarsonemus spinki Smiley), se encuentra ampliamente distribuido en Asia Tropical, además se reporta en República Dominicana, Haití, Cuba y Costa Rica. En Asia, ha ocasionado pérdidas en los rendimientos de hasta un $60 \%$ y en Cuba hasta un $80 \%$ (Ciencia y Tecnología, 2009).

La duración del ciclo de vida, en condiciones de laboratorio, varía entre 5 y 9 días. La mayor población de la plaga se encontró en las vainas de las hojas 2 y 3 (vainas inferiores), mientras que la población de los depredadores se distribuye de manera general en las mismas vainas (Ramos y Rodríguez 2001). La fenología del cultivo, la humedad relativa y la temperatura son factores que influyen significativamente sobre la densidad de S. spinki.

Este ácaro ocasiona dos tipos de daño al arroz: 1. Directo, ya que al alimentarse 
extrae el contenido de la vaina de las hojas induciendo a la deshidratación y la muerte del tejido. Además, se alimenta de los granos en formación, impidiendo su llenado, lo que es conocido como vaneo (Sanabria et al. 2004). 2. Indirecto, porque durante su alimentación, el ácaro inyecta sustancias tóxicas a las células vegetales para mantener el contenido celular fluyendo sin obstáculos; estas toxinas provocan deformaciones en el tejido vegetal, especialmente en el grano, induciendo a lo que se conoce como grano "pico de lora" (Sanabria et al. 2004).

Por ser una plaga de reciente introducción en nuestro país, no se han realizado estudios de su combate, de la tolerancia de variedades, tipos de muestreo entre otros. Por ello, este experimento se hizo con el fin de buscar variedades o líneas (León 2004), que sean menos o del todo no preferidas por el ácaro e incorporarlas en el programa de mejoramiento genético y de control integrado del ácaro, sin causar daños al ambiente, la salud humana y la calidad del grano.

Actualmente, las variedades cultivadas más comunes de arroz en Costa Rica son: CR-1113, CR-5272, CR-1821, CR-4477, FEDEARROZ 50, SENUMISA-02, SENUMISA-03, SENUMISA-04, CR-4338, CR-4102, CFX18 (Clearfield), Coprosen, Setesa 9.

Cada una de estas variedades posee sus características fenotípicas y genotípicas que las hacen preferidas por los agricultores, así como por la producción y resistencia a plagas y enfermedades por ejemplo, CR1113 se caracteriza por ser una variedad de paja corta, de hojas erectas, tallos gruesos, de buen macollamiento, resistente al acame, que florece entre 95 - 100 días después de la siembra, con un ciclo de producción a la cosecha entre 127 - 135 días, es resistente a la mayoría de las enfermedades que afectan el cultivo, pero susceptible a piricularia (MAG 1991) y al ácaro (León 2004), mientras que CR-5272 es una variedad de porte bajo, hojas erectas, macollamiento moderado, resistente al acame, con floración entre los 80 - 85 días después de la siembra y una duración a la cosecha entre 110 y 115 días (MAG 1991).

El objetivo de este estudio fue evaluar diferentes germoplasmas de arroz para determinar el grado de reacción al ácaro Steneotarsonemus spinki, en dos zonas arroceras de Costa Rica.

\section{MATERIALES Y MÉTODOS}

El estudio se realizó en dos regiones del país, en la Región Chorotega, específicamente en la Estación Experimental Enrique Jiménez Núñez ubicada en Taboga, Cañas, Guanacaste (Lat: 1020'N Log: 8510'O) a una altura de $20 \mathrm{msnm}$, con una temperatura promedio de $27 \stackrel{\circ}{\circ}$, $85 \%$ humedad relativa, la precipitación de 152,86 mm, humedad relativa de $77,67 \%$, la zona de vida es bosque tropical seco. El tipo de suelo es arcilloso, tipo molisoles, muestra un $\mathrm{pH}$ de 6,4. En la Región Pacífico Central en la Finca La DAPASA ubicada en Parrita, Puntarenas (Lat: 0930'N Long: 84을 $20^{\prime} \mathrm{O}$ ), con una altitud de $10 \mathrm{msnm}$, un promedio de $328 \mathrm{~mm}$ de lluvia caída, $27{ }^{\circ} \mathrm{C}$ de temperatura y $77 \%$ de humedad relativa, datos que corresponden al año 2004, se encuentra en la zona de vida, bosque tropical húmedo. Con dos tipos de material genotípico: Variedades criollas de autoconsumo o de refrescamiento (semilla que ha perdido vigor) con el objetivo de conservar en el banco de germoplasma del programa y Líneas promisorias, que son materiales introducidos promisorios los cuales se sembraron en parcelas de observación cuyo objetivo fue, evaluar a nivel nacional material genético altamente promisorio, que será utilizado posteriormente como materiales de rendimiento en experimentos regionales, como un paso previo a la liberación de nuevas variedades.

\section{VARIEDADES CRIOLLAS}

El trabajo de variedades criollas se sembró el día 18 de junio del año 2004. Utilizando las siguientes 24 variedades: Nira blanca, Tenorio, Gallote, Oryzica turipana, Cola del diablo, Miravalles, Nira colorado, Santa Rosa, Blue Bonnet, Chin Chin, Villano, Mata de plátano, Rex, Fedearroz 50, Senumisa 02 y CR-4477. 
La primera evaluación se realizo cuando el cultivo tenía 48 días de edad, la segunda a los 63 días, la tercera a los 77 días y la última a los 98 días después de la siembra. Se hicieron un total de seis evaluaciones, pero en la primera evaluación que fue a los 28 días y en la quinta evaluación, las muestras no mostraron presencia del ácaro en las vainas de las diferentes variedades.

\section{LINEAS PROMISORIAS}

Las líneas promisorias se sembraron en la Estación Experimental Enrique Jiménez Nuñez (EEEJN) y en Parrita. Se sembraron 59 materiales promisorios o líneas el 12 de agosto del 2004 en la EEEJN (Estación Experimental Enrique Jiménez Núñez), los datos aparecen en el Cuadro 1. Durante el ciclo fenológico del cultivo para determinar las densidades del ácaro se realizaron dos evaluaciones a los 22 días y 43 días después de la siembra.

Cuadro 1. Número de parcelas sembradas con el material promisorio a evaluar. Estación Experimental Enrique Jiménez Núñez. Guanacaste, Costa Rica. 12 de agosto, 2004.

\begin{tabular}{|c|c|c|c|}
\hline № parcela & Líneas/ variedades & № parcela & Líneas/ variedades \\
\hline 1 & CT15675-7-1-7-2-1-M & 30 & CT15765-13-7-2-1-2-M \\
\hline 2 & CT15675-7-1-4-1-1-M & 31 & CT15765-13-3-8-3-3-M \\
\hline 3 & CT15671-1-15-1-4-2-M & 32 & CT15671-15-1-3-1-M \\
\hline 4 & CT15675-7-1-7-1-2-M & 33 & CT15672-2-2-5-1-2-M \\
\hline 5 & CT15675-7-1-4-3-1-M & 34 & CT15671-15-1-7-1-1-M \\
\hline 6 & CT15671-15-1-4-2-4-M & 35 & CT15671-15-4-5-2-1-M \\
\hline 7 & CT15765-13-3-8-2-2-M & 37 & CT15671-16-1-7-1-1-M \\
\hline 8 & CT15675-7-1-7-3-3-M & 38 & CT15696-3-3-5-1-1-M \\
\hline 9 & CT15671-16-1-7-1-2-M & 39 & CT15672-12-1-1-2-1-M \\
\hline 10 & CT15675-2-2-2-1-1-M & 40 & CR-1113 \\
\hline 11 & CT15765-18-1-5-2-2-M & 41 & FEDEARROZ-50 \\
\hline 12 & CT15671-15-1-7-4-1-M & 42 & CR-4477 \\
\hline 13 & CT15691-4-5-2-2-4-M & 43 & CT15672-12-1-2-1-3-M \\
\hline 14 & CT15671-15-4-5-1-1-M & 44 & CT15765-12-1-4-2-1-M \\
\hline 15 & CT15672-12-1-5-2-2-M & 45 & CT15672-12-1-5-3-4-M \\
\hline 16 & CT15724-13-3-6-2-1-M & 46 & CT15675-7-1-7-3-2-M \\
\hline 17 & CT15765-13-3-6-1-2-M & 47 & CT15765-13-7-4-2-1-M \\
\hline 18 & CT15671-15-4-5-2-2-M & 48 & CT15672-2-2-5-1-1-M \\
\hline 19 & CT15671-15-4-2-2-2-M & 49 & CT15765-13-7-4-1-2-M \\
\hline 20 & CR-1113 & 50 & CT15671-15-4-6-2-3-M \\
\hline 21 & CR-4477 & 51 & CT15765-18-1-5-3-2-M \\
\hline 22 & FEDEARROZ-50 & 52 & CT15765-18-1-5-3-2-M \\
\hline 23 & CT15671-15-1-7-3-4-M & 53 & CT15672-12-1-2-1-1-M \\
\hline 24 & CT15671-15-4-7-1-1-M & 54 & CT15691-4-5-2-2-1-M \\
\hline 25 & CT15765-13-3-6-1-1-M & 55 & CT15675-7-1-4-2-1-M \\
\hline 26 & CT15672-12-1-1-2-3-M & 56 & CT15675-7-3-3-3-1-M \\
\hline 27 & CT15675-2-2-3-1-2-M & 57 & CR-1113 \\
\hline 28 & CT15675-7-1-4-2-3-M & 58 & FDEARROZ-50 \\
\hline 29 & CT15673-8-7-1-4-M & 59 & CR-4477 \\
\hline
\end{tabular}


Las líneas promisorias se sembraron en Parrita el día 14 de junio del 2004, corresponden al cuadro 2. Se realizaron tres evaluaciones, la primera a los 76 días, la segunda a los 86 días y la última a los 107 días después de la siembra.

Cuadro 2. Número de parcela sembrada con el material promisorio a evaluar. Parrita, Puntarenas, Costa Rica. 14 de junio, 2004.

\begin{tabular}{|c|c|c|c|}
\hline № parcela & Líneas/ variedades & № parcela & Líneas/ variedades \\
\hline 1 & CT15672-12-1-1-2-1-M & 34 & CT15765-18-1-5-2-2-M \\
\hline 2 & CT15675-7-1-7-3-2-M & 35 & CT15671-15-4-2-2-2-M \\
\hline 3 & CT15671-15-1-7-4-1-M & 36 & CT15671-16-1-7-1-2-M \\
\hline 4 & CT15675-2-2-2-1-1-M & 37 & CT15673-8-4-7-1-4-M \\
\hline 5 & CT15671-15-4-5-2-1-M & 38 & CT15765-13-7-2-1-2-M \\
\hline 6 & CT15671-15-4-6-2-3-M & 39 & CT15675-7-1-7-2-1-M \\
\hline 7 & CT15671-15-1-4-2-4-M & 40 & CR-5272 \\
\hline 8 & CT15765-13-3-6-2-1-M & 41 & CR-4477 \\
\hline 9 & CT15672-12-1-2-1-1-M & 42 & FEDEARROZ-50 \\
\hline 10 & CR-5272 & 43 & CT15675-03-3-1-M \\
\hline 11 & CR-4477 & 44 & CT15671-15-4-5-1-1-M \\
\hline 12 & FEDEARROZ-50 & 45 & CT15765-18-1-5-3-2-M \\
\hline 13 & CT15675-7-1-4-1-1-M & 46 & CT15671-15-1-7-3-4-M \\
\hline 14 & CT15765-12-1-4-2-1-M & 47 & CT15672-2-2-5-1-2-M \\
\hline 15 & CT15675-2-2-3-1-2-M & 48 & CT15672-12-1-5-3-4-M \\
\hline 16 & CT15765-13-3-8-3-3-M & 49 & CT15675-7-1-7-3-3-M \\
\hline 17 & CT15672-12-1-5-2-2-M & 50 & CR-5272 \\
\hline 18 & CT15765-13-3-8-2-2-M & 51 & CR-4477 \\
\hline 19 & CT15671-15-4-5-2-2-M & 52 & FDEARROZ-50 \\
\hline 20 & CR-5272 & 53 & CT15672-12-1-2-1-3-M \\
\hline 21 & CR-4477 & 54 & CT15671-15-1-3-1-3-M \\
\hline 22 & FEDEARROZ-50 & 55 & CT15671-16-1-7-1-1-M \\
\hline 23 & CT15765-13-7-4-1-2-M & 56 & CT15675-7-1-7-1-2-M \\
\hline 24 & CT15675-7-1-4-3-1-M & 57 & CT15765-13-7-4-2-1-M \\
\hline 25 & CT15691-4-5-2-2-4-M & 58 & CT15672-12-1-5-2-4-M \\
\hline 26 & CT15671-15-1-4-2-2-M & 59 & CT15671-15-4-7-2-2-M \\
\hline 27 & CT15671-15-1-4-3-3-M & 60 & CR-5272 \\
\hline 28 & CT15675-7-1-4-2-3-M & 61 & CR-4477 \\
\hline 29 & CT15672-2-2-5-1-1-M & 62 & FDEARROZ-50 \\
\hline 30 & CR-5272 & 63 & CT15672-12-1-1-2-3-M \\
\hline 31 & CR-4477 & 64 & CT15691-4-5-2-2-1-M \\
\hline 32 & FEDEARROZ-50 & 65 & CT15675-7-1-4-2-1-M \\
\hline 33 & CT15671-15-4-7-1-1-M & 66 & CT15696-3-3-5-1-1-M \\
\hline
\end{tabular}


El tamaño de las parcelas en todos los trabajos fue de $10 \mathrm{~m} 2$. El muestreo se realizó tomando tres plantas al azar de cada parcela por variedad. Las vainas de las hojas fueron separadas de los tallos de donde se revisaron al estereoscopio con luz arriba y abajo y hasta $40 \mathrm{X}$ de aumento, se les contó los ácaros (huevos, larvas-ninfas y adultos) de la porción 1 (base), 2 (centro) y 3 (collar) de cada hoja. La vaina se separa de la hoja y se divide en tres porciones como se aprecia en la Figura 1.

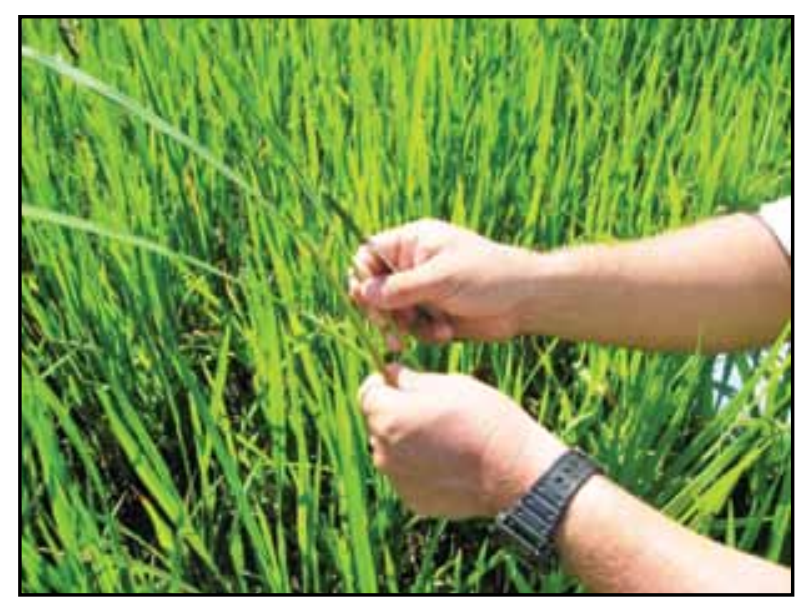

Figura 1. La vaina se separa de la hoja y se divide en tres porciones (base, centro y collar)

Se utilizó un diseño de bloques completos al azar. Los datos obtenidos durante los muestreos de variedades criollas fueron sometidos a un análisis de varianza y los promedios de cada variable se compararon mediante la prueba de Duncan al $5 \%$. Se calculó el promedio de ácaros por vaina, como indicadores de la preferencia del ácaro por las mismas.

Además, los resultados se agruparon en una escala, la cual fue elaborada de acuerdo a la población total de ácaros presentes en las vainas durante todas las evaluaciones. De ahí que, se formaron cuatro grupos con su respectiva escala y descripción de la misma como se aprecia en el Cuadro 3.

Cuadro 3. Descripción de la escala elaborada para la población de ácaros, encontrados en las vainas de arroz de secano favorecido, durante el año 2004.

\begin{tabular}{ccc}
\hline No de grupo & Escala & Descripción de la escala \\
\hline 1 & 0 & Sin la presencia de ningún estado del ácaro \\
\hline 2 & $1-20$ & $\begin{array}{r}\text { De uno a veinte, de cualquiera de las formas } \\
\text { (Huevos, inmaduros, adultos) del ácaro }\end{array}$ \\
\hline 3 & $21-100$ & De veintiuno a cien, de cualquiera de las formas del ácaro \\
\hline 4 & $>100$ & De más de cien, de cualquiera de las formas del ácaro
\end{tabular}

Elaborada por León, R. 2004. 


\section{RESULTADOS Y DISCUSIÓN}

\section{Variedades criollas}

El comportamiento de S. spinkien las variedades se muestran en el Cuadro 4; se indica la población total encontrada en las cuatro evaluaciones que se realizaron en las diferentes variedades. La variedad preferida por el ácaro es la Chin Chin, la que presentó las más altas poblaciones de huevos, inmaduros y adultos.

Cuadro 4. Variedades criollas y población de los diferentes estados del ácaro, Estación Experimental Enrique Jiménez Núñez. Taboga, Cañas, Guanacaste. 2004.

\begin{tabular}{|c|c|c|c|c|c|}
\hline \multicolumn{3}{|c|}{ Región Chorotega } & \multicolumn{3}{|c|}{ Número de ácaros totales } \\
\hline № parcela & Variedad & Origen o procedencia & Huevos & Inmaduros & Adultos \\
\hline 1 & Nira blanca & |RRI & 154 & 48 & 40 \\
\hline 2 & Tenorio & CIAT & 7 & 0 & 6 \\
\hline 3 & Gallote & IDIAP Panamá & 0 & 0 & 2 \\
\hline 4 & Oryzica turipana & ICA Colombia & 0 & 0 & 0 \\
\hline 5 & Cola del diablo & IDIAP Panamá & 121 & 45 & 110 \\
\hline 6 & Miravalles & CIAT & 0 & 0 & 0 \\
\hline 7 & Nira colorado & IRRI & 1 & 1 & 2 \\
\hline 8 & Santa Rosa & IDIAP Panamá & 110 & 7 & 21 \\
\hline 9 & Blue Bonnet & CIAT-USA & 0 & 0 & 0 \\
\hline 10 & Chin Chin & SURIMAN & 275 & 159 & 192 \\
\hline 11 & Villano & IDIAP Panamá & 190 & 77 & 55 \\
\hline 12 & Mata de plátano & Criollo Programa Arroz C.R. & 54 & 32 & 66 \\
\hline 13 & Rex Oro & CIAT-USA & 309 & 41 & 53 \\
\hline 14 & Costeño & IDIAP Panamá & 18 & 13 & 11 \\
\hline 15 & Orosí & Criollo CR & 2 & 1 & 0 \\
\hline 16 & CR-1113 & CIAT & 6 & 1 & 36 \\
\hline 17 & CR-4102 & CIAT & 90 & 77 & 48 \\
\hline 18 & CR-1821 & CIAT & 0 & 0 & 0 \\
\hline 19 & CR-5272 & CIAT & 23 & 13 & 18 \\
\hline 20 & CR-4338 & CIAT & 41 & 7 & 36 \\
\hline 21 & Senumisa 04 & Empresa Privada Colombia & 42 & 22 & 57 \\
\hline 22 & Fedearroz 50 & FEDEARROZ Colombia & 281 & 80 & 171 \\
\hline 23 & Senumisa 02 & FEDEARROZ Colombia & 25 & 13 & 23 \\
\hline 24 & CR-4477 & CIAT & 0 & 0 & 0 \\
\hline
\end{tabular}

Con los resultados de la separación de medias según Duncan al $5 \%$, se elaboró una agrupación de los materiales. Dicha agrupación indicó la formación de tres grupos o escalas de preferencia al ácaro, según la variedad del cultivo. En el Cuadro 5, se muestra la cantidad total de: huevos, inmaduros y adultos en cada una de las variedades. 
Cuadro 5. Promedio de Huevos, Inmaduros y Adultos por vaina, de acuerdo a separación de medias (Duncan al $5 \%$ ) según variedad. E.E.E.J.N., Cañas, Guanacaste, Costa Rica. 2004.

\begin{tabular}{|c|c|c|}
\hline $\begin{array}{c}\text { Variedades } \\
\text { (Huevos /vaina) }\end{array}$ & $\begin{array}{c}\text { Variedades } \\
\text { (Inmaduros/ vaina) }\end{array}$ & $\begin{array}{c}\text { Variedades } \\
\text { (adultos/ vaina) }\end{array}$ \\
\hline Datos & Datos & Datos \\
\hline transformados a & transformados a & transformados a \\
\hline raíz de $x+1, R^{2}=$ & raíz de $x+1$ & raíz de $x+1$ \\
\hline $25 \% C V=82,5$ & $R^{2}=21 \% C V=55,47$ & $R^{2}=22,35 \% C V=64,09$ \\
\hline Chin Chin a & Chin Chin a & Chin Chin a \\
\hline Rex Oro a & Villano ab & Nira Blanco abcd \\
\hline Nira blanco abcde & Rex Oro ab & Santa Rosa abcd \\
\hline Santa Rosa abcde & Nira Colorado & Villano abcd \\
\hline Villano abcde & Santa Rosa ab & Mata de Plátano abcd \\
\hline Mata de plátano abcde & Mata de Plátano ab & Rex Oro abcd \\
\hline Costeño abcde & Costeño ab & Costeño abcd \\
\hline CR-4102 abcde & Nira Blanco ab & CR1113 abcd \\
\hline CR-1113 abcde & Fedearroz-50 ab & CR-4102 abcd \\
\hline CR-1821 abcde & CR-5272 ab & CR1821 abcd \\
\hline CR-4338 abcde & CR-4338 ab & CR-5272 abcd \\
\hline Senumisa 02 abcde & Senumisa $02 a b$ & CR4338 abcd \\
\hline Senumisa 04 abcde & Senumisa 04 ab & Senumisa 04 abcd \\
\hline Fedearroz-50 abcde & Tenorio a & Fedearroz-50 abcd \\
\hline CR-5272 abcde & Gallote a & Senumisa 02 abcd \\
\hline Oryzica turipana e & Orosí a & Gallote d \\
\hline Cola del diablo e & Oryzica turipana a & Oryzica turipana d \\
\hline Miravalles e & Cola del diablo a & Cola del Diablo d \\
\hline Blue Bonnet e & Miravalles a & Miravalles d \\
\hline Orosí e & Blue Bonnet a & Nira Colorado d \\
\hline CR-4477 e & CR-1113 a & Blue Bonnet d \\
\hline Tenorio e & CR-4477 a & Orosí d \\
\hline Nira colorado e & CR-4102 ab & CR-4477 d \\
\hline Gallote e & CR-1821 a & Tenorio d \\
\hline
\end{tabular}


Los datos de la cantidad de huevos por vaina, forman tres grupos, el grupo a, que alcanzó el mayor porcentaje con 1,69 huevos/ vaina fue la Rex Oro y Chin Chin, luego el grupo abcde, con un porcentaje entre 1,02 a 1,64 \% huevos/ vaina lo obtuvieron las variedades Nira blanco, Santa Rosa, Villano, Mata de Plátano, Costeño, CR-4102, CR-1113, CR-1821, CR-4338, SENUMISA 02, SENUMISA 04, y Fedearroz 50 y el grupo e, que no presentó huevos en sus vainas corresponden a las variedades Gallote, Oryzica turipana, Cola del Diablo, Miravalles, Blue Bonnet, Orosí y CR-4477.

Las variedades Nira Blanca, Cola de Diablo, Santa Rosa, Chin Chin, Villano y Rex Oro, presentaron más de 100 huevos en los tres muestreos, por lo que estas variedades no se podrán considerar para mejoramiento genético.

El porcentaje de ácaros en estados inmaduros del grupo a, cuenta con 1,52 \% inmaduros/ vaina, que corresponde a la variedad Chin Chin, el grupo ab alcanzó desde 1,01 a 1,32 inmaduros/ vaina y el grupo b no presentó inmaduros en sus vainas. De las 24 variedades 17 presentaron inmaduros y siete durante los muestreos no presentaron este estadio del ácaro.

El porcentaje de adultos del grupo a cuenta con $1,74 \%$ de adultos/vainas, que corresponde a la variedad Chin Chin, el grupo abcd alcanzó desde 1,02 a 1,47 inmaduros/ vaina y en el grupo $d$ no se obtuvo adultos en sus vainas, Cuadro 5.

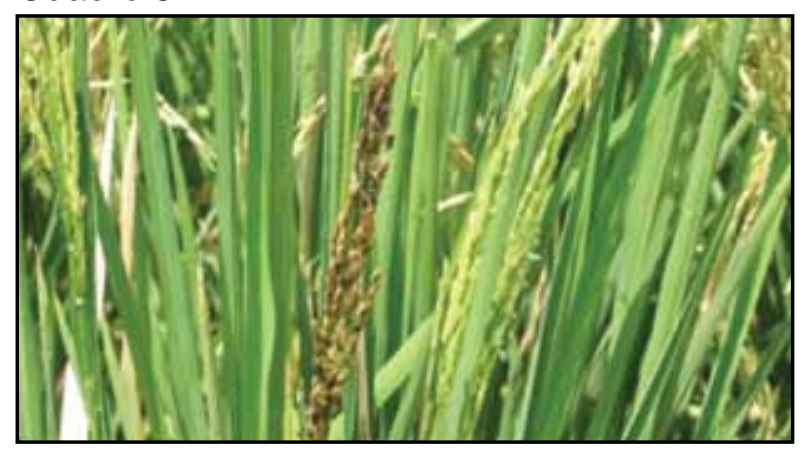

Figura 2. Daños en panícula, atribuidos al ácaro S. spinki. Taboga, Cañas, Guanacaste, Costa Rica. 2004.
La Figura 2 muestra una panícula erecta por estar los granos vanos, también los granos presentan manchado, estos síntomas se relacionan con el ácaro.

El Cuadro 6, agrupa de acuerdo a las poblaciones alcanzadas, de todos los estados del ácaro la preferencia o no del arácnido, a las diferentes variedades. Comparando esta escala con los tres grupos que se forman según Duncan al $5 \%$, la escala es más sencilla para interpretarla.

Cuadro 6. Escala o grupos de preferencia de acuerdo al total de la población de ácaros/ planta (huevos, inmaduros, adultos) en las diferentes variedades. Cañas, Guanacaste. Junio-Diciembre, 2004.

\begin{tabular}{|c|c|c|c|}
\hline $\mathbf{N}^{\circ}$ de & Escala & Descripción & Variedades \\
\hline 1 & 0 & $\begin{array}{c}\text { Sin la presencia } \\
\text { de ningún estado } \\
\text { del ácaro }\end{array}$ & $\begin{array}{c}\text { Oryzica turipana, } \\
\text { Miravalles, Blue } \\
\text { Bonnet, CR-1821, } \\
\text { y CR-4477. }\end{array}$ \\
\hline 2 & $1-20$ & $\begin{array}{l}\text { De uno a veinte, } \\
\text { de cualquiera de } \\
\text { las formas } \\
\text { (huevos, } \\
\text { inmaduros, } \\
\text { adultos) del ácaro }\end{array}$ & $\begin{array}{l}\text { Tenorio, Gallote, } \\
\text { Nira Colorado, } \\
\text { Costeño y Orosí. }\end{array}$ \\
\hline 3 & $21-100$ & $\begin{array}{l}\text { De veintiuno a } \\
\text { cien, de } \\
\text { cualquiera de } \\
\text { las formas del } \\
\text { ácaro }\end{array}$ & $\begin{array}{c}\text { Mata plátano, } \\
\text { CR-1113, } \\
\text { CR-4102, } \\
\text { CR-5272, } \\
\text { CR-4338, } \\
\text { Senumisa 04 y } \\
\text { Senumisa } 02\end{array}$ \\
\hline \multirow[t]{2}{*}{4} & $>100$ & $\begin{array}{l}\text { Más de cien, } \\
\text { de cualquiera } \\
\text { de las formas } \\
\text { del ácaro }\end{array}$ & $\begin{array}{l}\text { Nira Blanco, Cola } \\
\text { del Diablo, Santa } \\
\text { Rosa, Chin Chin, } \\
\text { Villano, Rex Oro y }\end{array}$ \\
\hline & & & Fedearroz-50 \\
\hline
\end{tabular}

Según Santos et al. (2001), S. spinki es específico para el cultivo del arroz y que se requiere de un ácaro en la plantación para que se considere infestada. Sin embargo, se puede especular, basado en los resultados obtenidos en este estudio, que de un ácaro a veinte los daños al cultivo serían poco significativos si los comparamos con variedades susceptibles que tienen más de 21 ácaros en sus plantas y más todavía con aquellas que cuentan con 
poblaciones mayores a los 100 ácaros. Para concretar esta escala sería interesante valorar el rendimiento de cada una de las variedades basados en la población que obtuvo cada uno de los materiales.

Se agruparon el número de huevos, inmaduros y adultos durante todo el proceso de evaluaciones. La variedad con más huevos fue Rex Oro (309) seguida de Chin Chin (275) y las que no presentaron huevos fueron las variedades Oryzica turipana, Miravalles, Blue Bonnet, CR-1821 y CR-4477. En cuanto a inmaduros la variedad más susceptible fue Chin Chin y a los que no se les encontró ácaros en sus vainas fueron Oryzica turipana, Miravalles, Blue Bonnet, CR-1821, CR-4477, Tenorio y Gallote. En cuanto a la presencia de adultos la más susceptible fue Chin Chin y las que no se infestaron fueron Oryzica turipana, Miravalles, Blue Bonnet, CR-1821, CR-4477 y la Orosí. Por lo tanto, las variedades más susceptible al ácaro fueron la Chin Chin y Rex Oro con un total de 626 y 403 respectivamente de todos los estados del ácaro). Lo cual se muestra por separado en las Figuras 3, 4 y 5.

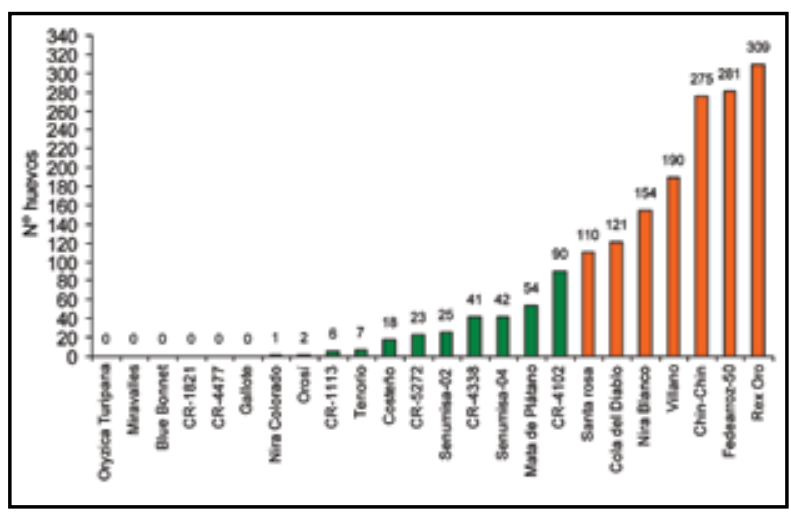

Figura 3. Total de huevos de $\boldsymbol{S}$. spinki según variedad. Taboga, Cañas, Guanacaste, Costa Rica. 2004.

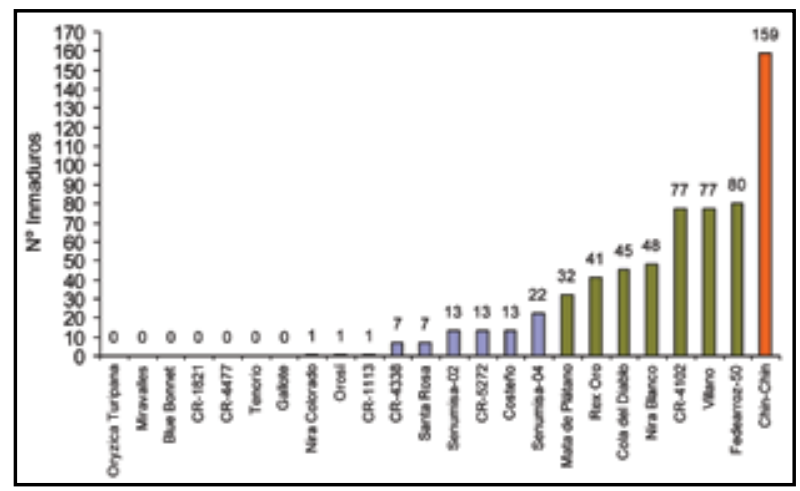

Figura 4. Total de inmaduros de $S$. spinki según variedad. Taboga, Cañas, Guanacaste, Costa Rica. 2004.

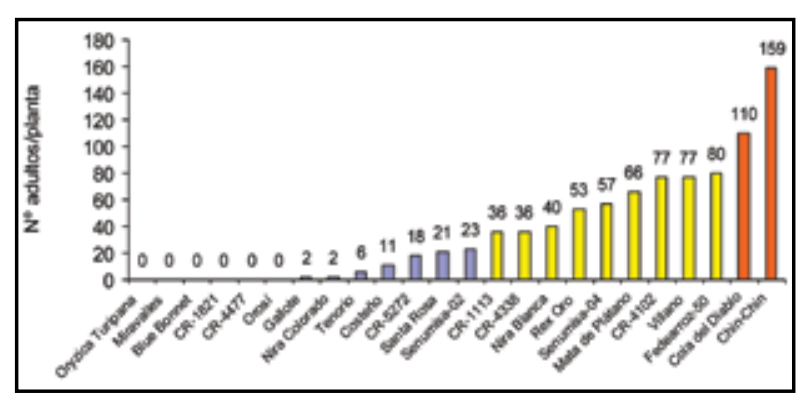

Figura 5. Total de adultos de $S$. spinki según variedad. Taboga, Cañas, Guanacaste, Costa Rica. 2004.

Como un esfuerzo por entender sobre la etapa fenológica en que afecta el ácaro al cultivo, se elaboró con las variedades criollas la Figura 6 , en ella se aprecia que a medida que se desarrollo el cultivo las poblaciones aumentaron pero a los 77 ddg la mayoría de las variedades aumentaron las poblaciones precisamente en la etapa que corresponde a la floración (Fase reproductiva). Esto podría deberse a que en este periodo se traslocan los azúcares y el almidón desde las vainas, hoja bandera, y vástagos, los cuales fueron acumulados en la fase vegetativa; situación que aprovecha el ácaros del vaneo del arroz para alimentarse y reproducirse. 


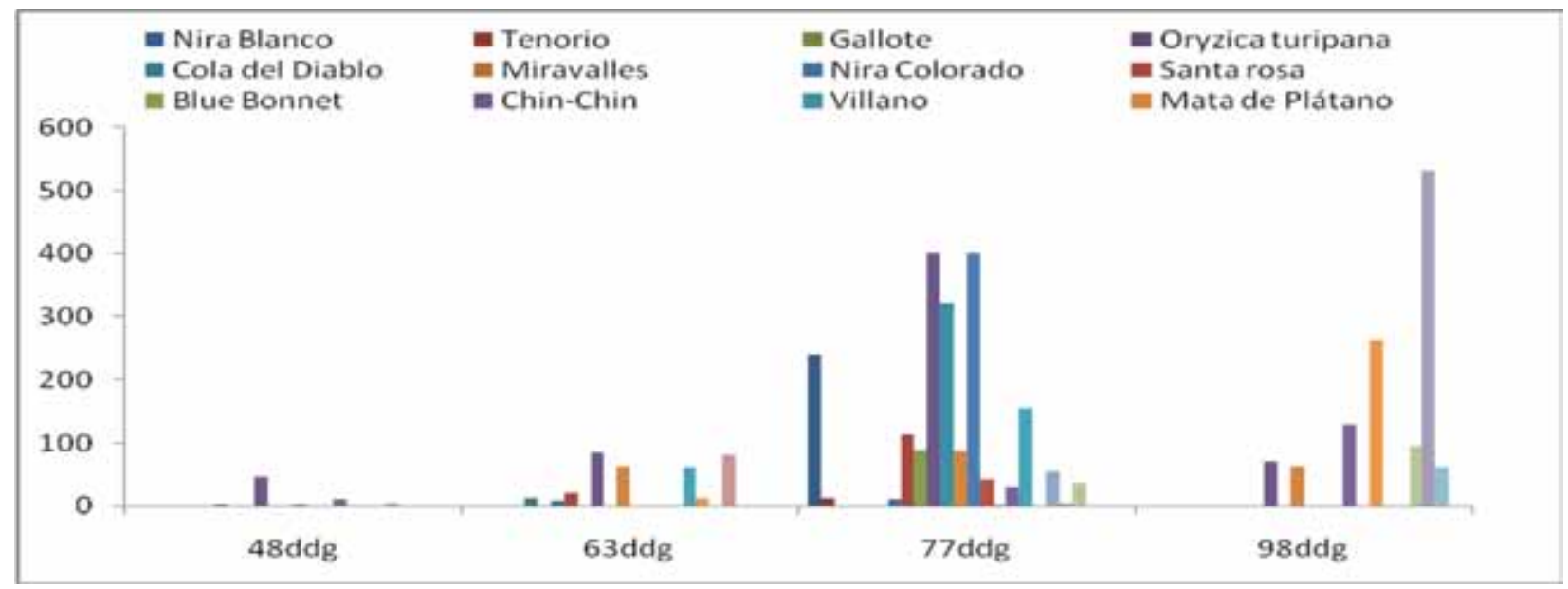

Figura 6. Población del ácaro de vaneo del arroz S. spinki. Días después de germinadas (ddg) las plantas de cada variedad. Taboga, Cañas, Guanacaste, Costa Rica. 2004.

Es interesante como la variedad Chin Chin desde inicio de la fase reproductiva (inicio de primordio floral) mantuvo las mayores poblaciones del ácaro y hasta la fase de maduración.

\section{LÍNEAS PROMISORIAS}

\section{En la Región Chorotega o Pacífico Seco, Taboga, Cañas}

Las líneas de mayor rendimiento en este lugar fueron la CT15671-15-4-5-1-1-M con 5,8 $\mathrm{t} /$ ha y se obtuvo un promedio de 278 ácaros/ planta (a/.p) , CT15672-12-1-5-2-4-M con 5,6 t/ ha y con 96 a/ p, CT15765-13-7-4-2-1-M con 4,7 t/ ha y 0 ácaros, CT15675-7-1-7-3-2-M con 2 ácaros/ planta y CT15672-12-1-1-2-3-M con 4,0 t/ ha con 0 a/ p. Al analizar los rendimientos con la infestación de ácaros se observó, que no hay correlación porque la línea más infestada fue la de mayor rendimiento. Este aspecto condujo a que en el campo no se hagan aplicaciones dirigidas al ácaro, sino más bien a la enfermedad fungo o bacterial que transmiten.

\section{En la Región Pacífico Central, Parrita, Finca Dapasa}

Los materiales de mayor rendimientos de campo fueron CT15675-7-1-4-2-1-M con 7,7 t/ ha, además no presentó durante las evaluaciones la presencia del ácaro, seguido de CT15765-181-5-3-2-M con 6,9 t/ ha, con cero ácaros, CT15672-12-1-2-1-1-M, con un promedio de 10,5 a/ p. 
ALCANCES TECNOLÓGICOS, AÑO 6, NÚMERO 1

Cuadro 7. Total de huevos, inmaduros y adultos del ácaro Steneotarsonemus spinki y su promedio de ácaros por planta en líneas y variedades evaluadas en Finca La Ligia en Parrita, Puntarenas, Costa Rica. 2004.

\begin{tabular}{|c|c|c|c|c|c|}
\hline \multicolumn{2}{|c|}{ Región Pacífico Central } & \multicolumn{4}{|c|}{ Parrita } \\
\hline \multirow[b]{2}{*}{ № parcela } & \multirow[b]{2}{*}{ Líneas/ variedades } & \multirow[b]{2}{*}{$\mathbf{H}$} & \multicolumn{2}{|c|}{ № ácaros totales } & \multirow[b]{2}{*}{ Prom/ planta } \\
\hline & & & I & A & \\
\hline 1 & CT15672-12-1-1-2-1-M & 0 & 0 & 0 & 0 \\
\hline 2 & CT15675-7-1-7-3-2-M & 0 & 0 & 0 & 0 \\
\hline 3 & CT15671-15-1-7-4-1-M & 0 & 0 & 0 & 0 \\
\hline 4 & CT15675-2-2-2-1-1-M & 0 & 0 & 0 & 0 \\
\hline 5 & CT15671-15-4-5-2-1-M & 0 & 0 & 0 & 0 \\
\hline 6 & CT15671-15-4-6-2-3-M & 10 & 0 & 30 & 13,30 \\
\hline 7 & CT15671-15-1-4-2-4-M & 0 & 0 & 0 & 0 \\
\hline 8 & CT15765-13-3-6-2-1-M & 55 & 12 & 42 & 36,30 \\
\hline 9 & CT15672-12-1-2-1-1-M & 0 & 0 & 0 & 0 \\
\hline 10 & CR-5272 & 15 & 7 & 26 & 16 \\
\hline 11 & CR-4477 & 0 & 0 & 0 & 0 \\
\hline 12 & FEDEARROZ-50 & 0 & 0 & 10 & 3,30 \\
\hline 13 & CT15675-7-1-4-1-1-M & 20 & 7 & 26 & 17,70 \\
\hline 14 & CT15765-12-1-4-2-1-M & 15 & 15 & 25 & 8,30 \\
\hline 15 & CT15675-2-2-3-1-2-M & 0 & 0 & 0 & 0 \\
\hline 16 & CT15765-13-3-8-3-3-M & 0 & 0 & 7 & 2,30 \\
\hline 17 & CT15672-12-1-5-2-2-M & 0 & 0 & 0 & 0 \\
\hline 18 & CT15765-13-3-8-2-2-M & 0 & 0 & 0 & 0 \\
\hline 19 & CT15671-15-4-5-2-2-M & 0 & 0 & 0 & 0 \\
\hline 20 & CR-5272 & 0 & 0 & 0 & 0 \\
\hline 21 & CR-4477 & 0 & 0 & 0 & 0 \\
\hline 22 & FEDEARROZ-50 & 0 & 0 & 0 & 0 \\
\hline 23 & CT15765-13-7-4-1-2-M & 10 & 5 & 65 & 31,70 \\
\hline 24 & CT15675-7-1-4-3-1-M & 0 & 0 & 0 & 0 \\
\hline 25 & CT15691-4-5-2-2-4-M & 5 & 70 & 25 & 33,30 \\
\hline 26 & CT15671-15-1-4-2-2-M & 0 & 0 & 0 & 0 \\
\hline 27 & CT15671-15-1-4-3-3-M & 0 & 0 & 0 & 0 \\
\hline 28 & CT15675-7-1-4-2-3-M & 0 & 0 & 5 & 1,70 \\
\hline 29 & CT15672-2-2-5-1-1-M & 0 & 0 & 0 & 0 \\
\hline 30 & CR-5272 & 0 & 0 & 6 & 2,0 \\
\hline 31 & CR-4477 & 0 & 0 & 0 & 0 \\
\hline
\end{tabular}


Evaluación Preliminar de Diferentes Germoplasmas de Arroz (Oryza Sativa) para Determinar la Preferencia del Ácaro Steneotarsonemus Spinki (Tarsonemidae) en Costa Rica.

\begin{tabular}{|c|c|c|c|c|c|}
\hline № parcela & Líneas/ variedades & $\mathbf{H}$ & $\mathrm{I}$ & A & Prom/ planta \\
\hline 32 & FEDEARROZ-50 & 0 & 0 & 0 & 0 \\
\hline 33 & CT15671-15-4-7-1-1-M & 0 & 0 & 0 & 0 \\
\hline 34 & CT15765-18-1-5-2-2-M & 0 & 0 & 0 & 0 \\
\hline 35 & CT15671-15-4-2-2-2-M & 0 & 0 & 0 & 0 \\
\hline 36 & CT15671-16-1-7-1-2-M & 0 & 0 & 0 & 0 \\
\hline 37 & CT15673-8-4-7-1-4-M & 0 & 0 & 6 & 2,00 \\
\hline 38 & CT15765-13-7-2-1-2-M & 0 & 0 & 27 & 9,00 \\
\hline 39 & CT15675-7-1-7-2-1-M & 6 & 0 & 0 & 2,00 \\
\hline 40 & CR-5272 & 3 & 1 & 13 & 5,70 \\
\hline 41 & CR-4477 & 0 & 0 & 0 & 0 \\
\hline 42 & FEDEARROZ-50 & 0 & 0 & 0 & 0 \\
\hline 43 & CT15675-03-3-1-M & 0 & 0 & 0 & 0 \\
\hline 44 & CT15671-15-4-5-1-1-M & 110 & 35 & 92 & 79 \\
\hline 45 & CT15765-18-1-5-3-2-M & 2 & 2 & 4 & 2,70 \\
\hline 46 & CT15671-15-1-7-3-4-M & 0 & 2 & 0 & 0,70 \\
\hline 47 & CT15672-2-2-5-1-2-M & 0 & 0 & 0 & 0 \\
\hline 48 & CT15672-12-1-5-3-4-M & 0 & 0 & 15 & 5 \\
\hline 49 & CT15675-7-1-7-3-3-M & 0 & 0 & 9 & 3 \\
\hline 50 & CR-5272 & 7 & 3 & 7 & 5,70 \\
\hline 51 & CR-4477 & 0 & 0 & 0 & 0 \\
\hline 52 & FDEARROZ-50 & 0 & 0 & 0 & 0 \\
\hline 53 & CT15672-12-1-2-1-3-M & 0 & 0 & 0 & 0 \\
\hline 54 & CT15671-15-1-3-1-3-M & 2 & 0 & 1 & 1 \\
\hline 55 & CT15671-16-1-7-1-1-M & 0 & 0 & 0 & 0 \\
\hline 56 & CT15675-7-1-7-1-2-M & 25 & 7 & 15 & 15,70 \\
\hline 57 & CT15765-13-7-4-2-1-M & 112 & 17 & 105 & 78 \\
\hline 58 & CT15672-12-1-5-2-4-M & 0 & 0 & 0 & 0 \\
\hline 59 & CT15671-15-4-7-2-2-M & 0 & 0 & 0 & 0 \\
\hline 60 & CR-5272 & 0 & 0 & 0 & 0 \\
\hline 61 & CR-4477 & 0 & 0 & 0 & 0 \\
\hline 62 & FDEARROZ-50 & 38 & 12 & 79 & 43 \\
\hline 63 & CT15672-12-1-1-2-3-M & 0 & 0 & 0 & 0 \\
\hline 64 & CT15691-4-5-2-2-1-M & 0 & 0 & 0 & 0 \\
\hline 65 & CT15675-7-1-4-2-1-M & 0 & 0 & 0 & 0 \\
\hline 66 & CT15696-3-3-5-1-1-M & 30 & 20 & 60 & 36,70 \\
\hline
\end{tabular}

CT $=$ CIAT $\quad 15.672=$ Material F6 $\quad M=$ Masal 
ALCANCES TECNOLÓGICOS, AÑO 6, NÚMERO 1

Cuadro 8. Total de todos los estados del ácaro y promedio de ácaros por planta en líneas y variedades evaluadas en Estación Experimental Enrique Jiménez Núñez. Taboga, Cañas, Guanacaste. 2004.

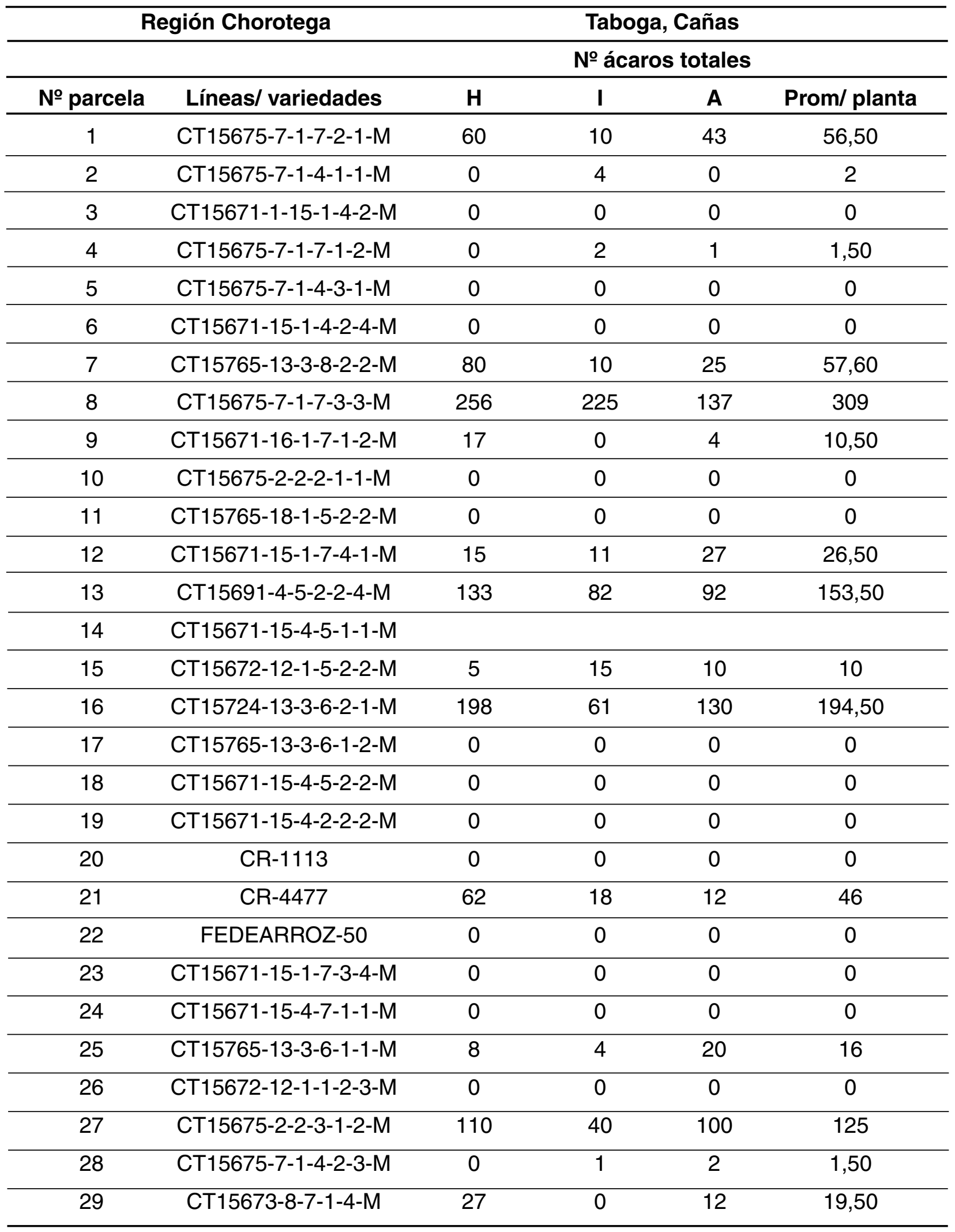


Evaluación Preliminar de Diferentes Germoplasmas de Arroz (Oryza Sativa) para Determinar la Preferencia del Ácaro Steneotarsonemus Spinki (Tarsonemidae) en Costa Rica.

\begin{tabular}{|c|c|c|c|c|c|}
\hline № parcela & Líneas/ variedades & $\mathbf{H}$ & $\mathbf{I}$ & $\mathbf{A}$ & Prom/ planta \\
\hline 30 & CT15765-13-7-2-1-2-M & 0 & 0 & 0 & 0 \\
\hline 31 & CT15765-13-3-8-3-3-M & 1 & 0 & 2 & 1,50 \\
\hline 32 & CT15671-15-1-3-1-M & 0 & 0 & 0 & 0 \\
\hline 33 & CT15672-2-2-5-1-2-M & 0 & 0 & 0 & 0 \\
\hline 34 & CT15671-15-1-7-1-1-M & 25 & 7 & 15 & 24,50 \\
\hline 35 & CT15671-15-4-5-2-1-M & 0 & 0 & 0 & 0 \\
\hline 36 & CT15672-12-1-5-2-4-M & 0 & 0 & 0 & 0 \\
\hline 37 & CT15671-16-1-7-1-1-M & 0 & 0 & 0 & 0 \\
\hline 38 & CT15696-3-3-5-1-1-M & 0 & 0 & 0 & 0 \\
\hline 39 & CT15672-12-1-1-2-1-M & 0 & 0 & 0 & 0 \\
\hline 40 & CR-1113 & 0 & 0 & 0 & 0 \\
\hline 41 & FEDEARROZ-50 & 0 & 0 & 0 & 0 \\
\hline 42 & CR-4477 & 7 & 11 & 10 & 14 \\
\hline 43 & CT15672-12-1-2-1-3-M & 0 & 0 & 0 & 0 \\
\hline 44 & CT15765-12-1-4-2-1-M & 410 & 48 & 98 & 278 \\
\hline 45 & CT15672-12-1-5-3-4-M & 0 & 0 & 0 & 0 \\
\hline 46 & CT15675-7-1-7-3-2-M & 0 & 0 & 0 & 0 \\
\hline 47 & CT15765-13-7-4-2-1-M & 0 & 0 & 0 & 0 \\
\hline 48 & CT15672-2-2-5-1-1-M & 0 & 0 & 0 & 0 \\
\hline 49 & CT15765-13-7-4-1-2-M & 0 & 0 & 0 & 0 \\
\hline 50 & CT15671-15-4-6-2-3-M & 0 & 0 & 0 & 0 \\
\hline 51 & CT15765-18-1-5-3-2-M & 0 & 0 & 0 & 0 \\
\hline 52 & CT15765-18-1-5-3-2-M & 92 & 0 & 2 & 47,02 \\
\hline 53 & CT15672-12-1-2-1-1-M & 0 & 0 & 0 & 0 \\
\hline 54 & CT15691-4-5-2-2-1-M & 0 & 0 & 0 & 0 \\
\hline 55 & CT15675-7-1-4-2-1-M & 0 & 0 & 0 & 0 \\
\hline 56 & CT15675-7-3-3-3-1-M & 85 & 40 & 70 & 97,50 \\
\hline 57 & CR-1113 & 0 & 0 & 0 & 0 \\
\hline 58 & FDEARROZ-50 & 90 & 40 & 62 & 96 \\
\hline 59 & CR-4477 & 0 & 0 & 0 & 0 \\
\hline
\end{tabular}

$\mathrm{CT}=\mathrm{CIAT} \quad 15.672=$ Material F6 $\mathrm{M}=$ Masal 
Las líneas promisorias que obtuvieron los mayores rendimientos fueron: la CT15671-15-4-5-1$1-\mathrm{M}$ con 5,8 t/ ha con un promedio de $278 \mathrm{a} / \mathrm{p}$, CT15672-12-1-5-2-4-M con 5,6 t/ ha y $96 \mathrm{a} / \mathrm{p}$, CT15765-13-7-4-2-1-M para un promedio de 4,7 t/ ha sin la presencia se ácaros, CT15675-7-17-3-2-M con 2 ácaros/ planta y finalmente la línea CT15672-12-1-1-2-3-M con 4,0 t/ ha con 0 a/ $p$. Estos resultados no muestran que las poblaciones mucha o poca afecte al rendimiento. Más bien se puede decir que no es la cantidad sino la etapa de desarrollo del cultivo el que influencia las poblaciones, así como el manejo del cultivo y el clima.

Al igual que en los resultados que se muestran en el Cuadro 6, se formó la misma escala con los resultados de los Cuadros 7 y 8 , lo que se resume en el Cuadro 9.

Cuadro 9. Escala o grupos de preferencia de acuerdo a la población de ácaros/ planta. Parrita y Taboga. Setiembre-Diciembre, 2004.

\begin{tabular}{|c|c|c|c|}
\hline$N^{\circ}$ de grupo & Escala & Descripción de la escala & Líneas/ variedades* \\
\hline 1 & $0-0$ & $\begin{array}{c}\text { Sin la presencia de ningún estado } \\
\text { del ácaro }\end{array}$ & $\begin{array}{c}3,5,17,18,19,24,26 \\
33,35,36,47,55,59 \\
63,64,65\end{array}$ \\
\hline 2 & $1-20$ & $\begin{array}{l}\text { Con la presencia de uno a } \\
\text { veinticinco, de cualquiera de las } \\
\text { formas (huevos, inmaduros, } \\
\text { adultos) del ácaro }\end{array}$ & $\begin{array}{c}2,4,6,9,10,11,12,14 \\
15,23,25,28,29,34,37 \\
38,39,43,45,46,48,49 \\
53,54,66\end{array}$ \\
\hline 3 & $21-100$ & $\begin{array}{c}\text { Con la presencia de } \\
\text { veintiséis a cien, de } \\
\text { cualquiera de las formas } \\
\text { del ácaro }\end{array}$ & $\begin{array}{c}1,7,13,16,27,56 \\
57,58\end{array}$ \\
\hline 4 & $>100$ & $\begin{array}{l}\text { Con la presencia de } \\
\text { más de cien, de cualquiera } \\
\text { de las formas del ácaro }\end{array}$ & 8,44 \\
\hline
\end{tabular}

*Número según parcela (Cuadros 7 y 8)

El grupo 0-0 corresponde a aquellas variedades que no presentaron ácaros en ninguno de sus estados, estas se pueden considerar para el programa de mejoramiento genético y estudiarlas con respecto a la relación ácaro-hongo-bacteria, mientras que las del grupo 1-20 ácaros, se debe analizar con respecto a la producción versus la presencia del ácaro. Luego las variedades con poblaciones mayores de 21 a 100 y más de 100 ácaros en las vainas, se deben evaluar con respecto a los rendimientos y la transmisión de enfermedades (relación ácaro-hongo-bacteria) y como afecta la población de ácaros en los rendimientos.

Se observaron diferencias del comportamiento de las variedades en las diferentes zonas agroclimáticas, tal es el caso de la variedad CR-1113, que no presentó ácaros en las condiciones de la Región Chorotega (E.E.E.J.N.), mientras la variedad CR-4477 si fue atacada por el ácaro y no así en las condiciones de la Región Pacífico Central (Parrita). El manejo también es un factor a considerar ya que las plantas sembradas en la E.E.E.J.N fueron manejadas con el objetivo de producir semilla de fundación, por lo que se le brindo los requerimientos de agua, nutricionales oportunamente y no se abuso del uso de plaguicidas. Mientras que en Parrita el manejo agronómico fue comercial y por lo tanto intensivo. Sin embargo esto no sucedió para todas las líneas estudiadas. 
Por lo que se aprecia en los resultados no se puede descartar materiales o variedades por el mero hecho de ser preferida o susceptible al ácaro del vaneo del arroz, ya que no se da una relación entre el rendimiento y la población obtenida en este estudio. Pero si es importante considerar la fase fenológica, el manejo agronómico y el clima con las poblaciones y el rendimiento del cultivo.

\section{CONCLUSIONES}

Las variedades criollas, que no mostraron ninguno de los estados del ácaro fueron, Oryzica turipana, Miravalles, Blue Bonnet y las variedades CR-1821 y CR-4477.

La variedad criolla, con mayor cantidad de ácaros en todos sus estados fue la Chin Chin.

La fenología y el manejo agronómico del cultivo son factores que influyen en las poblaciones del ácaro del vaneo del arroz.

Las líneas promisorias que obtuvieron los mayores rendimientos en las dos regiones de estudio fueron en la Región Chorotega fueron: la CT15671-15-4-5-1-1-M con 5,8 t/ ha con un promedio de 278 a/ p, CT15672-12-1-5-2-4-M con 5,6 t/ ha y $96 \mathrm{a} / \mathrm{p}$, CT15765-13-7-4-2-1-M para un promedio de $4,7 \mathrm{t} /$ ha sin la presencia de ácaros, CT15675-7-1-7-3-2-M con 2 ácaros/ planta y finalmente la línea CT15672-12-1-12-3-M con 4,0 t/ ha con 0 a/ p. En la Región Pacifico Central fueron CT15675-7-1-4-2-1-M con 7,7 t/ ha, sin la presencia de ninguno de los estados del ácaro, luego CT15765-18-1-53-2-M con 6,9 t/ ha, con cero ácaros, CT1567212-1-2-1-1-M, con un promedio de 10,5 a/ p.

La infestación de ácaros no correlaciona con rendimiento, porque el material (líneas) más infestada de ácaros fue la que obtuvo mayores rendimientos.

La presencia de huevos en las vainas, es un indicador de colonización o establecimiento del ácaro, en los diferentes germoplasmas de arroz.

\section{RECOMENDACIONES}

Se observó que todas las variedades o líneas que en este trabajo mostraron ser no hospedantes al ácaro, si son sembradas solas a nivel comercial, se afectan en mayor "escala" o son infestadas por el ácaro; pero si se combinan con una o más variedades o líneas susceptibles, los ácaros prefirieron las variedades o líneas susceptibles, esto puede servir para realizar aplicaciones dirigidas en las áreas de arroz susceptible.

Considerar en futuros experimentos, los problemas fitopatológicos en que el ácaro podría estar participando, como la relación de Sarocladium oryzae y Xanthomonas campestris, además del efecto en el rendimiento.

Para estudios de investigación es necesario buscar el lugar más cercano con el fin de colocar el estereoscopio y contar los ácaros en el lugar (in situ), no trasladar las plantas o vainas a lugares lejanos. El arroz se debe cortar en el momento de realizar el conteo.

Realizar análisis de contenidos de materia seca a las variedades que en las mismas condiciones tengan cero o menos de 20 ácaros/ planta. O tomar la variedad más susceptible en este caso Chin Chin con respecto a alguna que no se infestó con el ácaro.

Evaluar el daño en la producción de acuerdo a la población de ácaros en las variedades y a la infestación de hongos y bacterias.

Las variedades que no se emplean comercialmente, y muestran cierta tolerancia al ácaro, valdría la pena tomarlos en cuenta en mejoramiento genético.

\section{AGRADECIMIENTOS}

En forma muy especial a los funcionarios de la Estación Experimental Enrique Jiménez Núñez por su colaboración en facilitar el buen desarrollo de este estudio. 
Al Ing. Roberto Tinoco Mora, al Ing. Randoph Campos Morera (qdeDg), Ing. Juan C. Jiménez Vargas y al Tec. Rodrigo Dobles Gutiérrez (qdeDg), por sus valiosos aportes para la realización de este estudio (preparación del terreno, siembra, marcaje de parcelas y otros). A Carmen Hernández Ramírez por el conteo de los ácaros. Al Ing. Carlos Rodríguez Valverde por sus aportes en la revisión de este estudio.

\section{LITERATURA CITADA}

Ciencia y Tecnología. 2009. Comportamiento Poblacional de Steneotarsonemus Spinki Smiley (Acari: Tarsonemidae) en el Cultivo del Arroz (Oryza sativa L.). Temas de Ciencia y Tecnología 13(39): (septiembre diciembre) 55 - 66. Tomado de http:// mixteco.utm.mx/edi_anteriores/ Temas39/2NOTAS\%2039-4.pdf. Fecha: 18 marzo del 2010. Hora: 3:55 pm.

Araya, M. A. 2004. Manejo de plagas insectiles en el cultivo del arroz (Oryza sativa). Trabajo de presentación al curso de Entomología Agrícola. Universidad de Costa Rica. Turrialba. 23 p.

Alfonso, R.; Rodríguez, S. 2002. Comportamiento de líneas avanzadas de arroz de bajos insumos de agua y fertilizantes en la localidad de Bauta, La Habana. Instituto de Investigaciones del Arroz. La Habana, Cuba. 4 p.

Cochran, W. G.; Cox, G. M. 1965. Diseños experimentales. 2da ed. Editorial Trillas. México. p 618-644.

Food and Agriculture Organization (FAO). 2004. El arroz y la nutrición humana. Hoja divulgativa por el año internacional del arroz 2004, el arroz es vida. Disponible en www.rice2004. org. $2 p$.
León, G. R. 2004. Evaluación de la presencia del ácaro Steneotarsonemus spinki (Tarsonemidae) en diez variedades de arroz (Oryza sativa). INTA (Instituto Nacional de Innovación y Transferencia en Tecnología Agropecuaria-Ministerio de Agricultura y Ganadería) (Archivos técnicos) San José, Costa Rica. 6 p.

MAG (Ministerio de Agricultura y Ganadería) 1991. Aspectos técnicos sobre cuarenta y cinco cultivos agrícolas de Costa Rica. San José, Costa Rica. $557 \mathrm{p}$.

Miranda, I.; Ramos, M.; Fernández, B. 2003. Factores que influyen en la abundancia de Steneotarsonemus spinki en arroz, en Cuba. Manejo Integrado de Plagas y Agroecología (Costa Rica) (69): 34-37.

Ochoa, R. Aguilar, H. 1991. Ácaros fitófagos de América Central: Guía ilustrada. Manual Técnico № 6. CATIE (Centro Agronómico Tropical de Investigación y Enseñanza Turrialba). Turrialba, Costa Rica. 251 p.

Ramos, M.; Rodríguez, H. 2001. Aspectos biológicos de Steneotarsonemus spinki en arroz, en Cuba. Manejo Integrado de Plagas y Agroecología (Costa Rica) 61: 48-52.

Sanabria, C.; Aguilar, H. 2004. El ácaro del vaneo del arroz Steneotarsonemus spinkiSmiley. Ministerio de Agricultura y Ganadería. 16 p.

Santos, A.; Almaguel, L.; De la Torre, P.; Cáceres, I. 2001. Longevidad y fecundidad de Steneotarsonemus spinki (Acari: Tarsonemidae) en el cultivo del arroz en Cuba. Fitosanidad 5(3): 2. 